\title{
Implementation of an efficient dielectric function into the finite difference time domain method for simulating the coupling between localized surface plasmons of nanostructures
}

\author{
J.Y. Lu, Y.H. Chang* \\ Department of Physics, National Taiwan University, Taipei 106, Taiwan
}

\section{A R T I C L E I N F O}

\section{Article history:}

Available online $\mathrm{xxxx}$

\section{Keywords:}

FDTD

Dispersive materials

Localized surface plasmon

Core-shell nanostructure

\begin{abstract}
A B S T R A C T
An improved dielectric function was developed for the description of the dispersion of gold and silver in the wide spectral range between $180 \mathrm{~nm}$ and $2000 \mathrm{~nm}$. The dielectric function can be used in the finite difference time domain method more efficiently and accurately than the four-Lorentzian terms (L4) model. We use it to study the effect of the interaction between the silicacore gold-shell nanocylinder pair on the localized surface plasmon resonance spectra (LSPR) and the simulation result shows that for a polarization direction parallel to the axis of the pair, the plasmon resonance wavelength increases when the width of the gap between nanocylinders decreases.
\end{abstract}

(c) 2009 Elsevier Ltd. All rights reserved.

\section{Introduction}

In this work we use the finite difference time domain (FDTD) method to study the interaction between nanostructures. Comparing with other frequency domain electromagnetic simulation methods which can obtain solutions at a definite frequency, the FDTD method has the advantage that it can get the full spectrum in a single simulation by propagating a short pulse; but it requires a good analytical model for the description of the dispersion of materials. For the analytical model, the fourLorentzian (L4) model proposed by Hao and Norlander showed a better fit than other frequently-used dielectric functions for gold and silver in the spectral range between $180 \mathrm{~nm}$ and $2000 \mathrm{~nm}$ [1]. The other analytical model, known as the Drude critical point model which includes one Drude term and

\footnotetext{
* Corresponding author. Tel.: +886233665102.

E-mail address: yhchang@phys.ntu.edu.tw (Y.H. Chang).
} 
two critical point terms shows a better fitting quality than the L4 model in the near UV and visible region [2], but in the wide spectral range between $180 \mathrm{~nm}$ and $2000 \mathrm{~nm}$ the Drude critical point model is still less accurate than the L4 model overall.

In this paper, an improved dielectric function model is developed, together with a comparison of the improved model and L4 model with experimental data [3] and the fitting parameters for gold and silver are presented. The results show that the model we present is better than the L4 model for gold and silver, both in accuracy and computational efforts in the spectral range between $180 \mathrm{~nm}$ and $2000 \mathrm{~nm}$. We use it to simulate the interaction between a pair of core-shell nanocylinders for the polarization direction parallel to the axis of the pair. The results show that the plasmon resonance wavelength red-shifted with decreasing gap width.

\section{Dispersion models}

\subsection{The L4 model}

An analytical model including the four-Lorentzian terms (L4) has been recently presented for the description of the dispersion of gold and silver in the wide wavelength range between $180 \mathrm{~nm}$ and $2000 \mathrm{~nm}$. The model can be expressed as

$$
\varepsilon(\omega)=\varepsilon_{\infty}+\frac{\sigma / \varepsilon_{0}}{\mathrm{i} \omega}+\sum_{p=1}^{4} \frac{C_{p}}{\omega^{2}+A_{p} \mathrm{i} \omega+B_{p}} .
$$

We can implement the L4 model by recursive convolution (RC) method or auxiliary differential equation (ADE) method which can be derived from [4] into FDTD method with the fitting parameters given in [1]. Although this model is suitable for simulating highly tunable metallic nanostructures such as nanoshell and nanoring, we should note that having more Lorentzian terms increases the accuracy of the fitting quality, it also increases the memory needed in the FDTD simulation. In addition, the L4 model is not accurate enough to describe the permittivity of Ag due to large variations in the imaginary part of the permittivity as compared with the experimental data. For the above reasons, it is desirable to have an analytical model that can best describe the dispersion of materials accurately and cost the least amount of memory at the same time.

\subsection{The CP3 model}

In order to obtain such an analytical model, we try to use critical point terms to replace the Lorentzian terms of $\mathrm{L} 4$ model because the critical point term gives better description of the interband transition of noble metals. The result shows that we can get better fitting quality than the L4 model for gold and silver by just three critical point terms. The three critical point pole pairs (CP3) model can be expressed as

$$
\varepsilon(\omega)=\varepsilon_{\infty}+\frac{\sigma / \varepsilon_{0}}{\mathrm{i} \omega}+\sum_{p=1}^{3}\left(\frac{A_{p} \Omega_{p} \mathrm{e}^{\mathrm{i} \phi_{p}}}{\Omega_{p}-\omega-\mathrm{i} \Gamma_{p}}+\frac{A_{p} \Omega_{p} \mathrm{e}^{-\mathrm{i} \phi_{p}}}{\Omega_{p}+\omega+\mathrm{i} \Gamma_{p}}\right) .
$$

Since the Fourier-transformed critical point term was shown to have a similar form as the Lorentzian term in the time domain [5], we can implement the critical point term of the CP3 model into the FDTD method by the RC method or ADE method. In order to implement it conveniently, for the readers, we offer the numerical implementation of the CP3 model with the RC method in the Appendix.

\subsection{Comparison of the models}

In this section we compare the fitting quality of L4 model and CP3 model with tabulated values of gold and silver in [3]. The parameters of L4 model for gold and silver are taken from [1], and the parameters of CP3 model for gold and silver are displayed in Table 1. The comparisons show that we 
Table 1

The optimized parameters for the CP3 model for gold and silver in the spectrum between $180 \mathrm{~nm}$ and $2000 \mathrm{~nm}$ and the values of the fitness function of the CP3 model and L4 model.

\begin{tabular}{llc}
\hline CP3 & $\mathrm{Au}$ & $\mathrm{Ag}$ \\
\hline$\varepsilon_{\infty}$ & 1.1156 & 1.4783 \\
$\sigma$ & $4.24 \times 10^{16}$ & $1.32863 \times 10^{16}$ \\
$A_{1}(\mathrm{rad})$ & 0.5548 & 1.007 \\
$\left.\phi_{1}(\mathrm{rad} \mathrm{s})^{-1}\right)$ & 2.8463 & -0.9621 \\
$\Omega_{1}\left(.506 \times 10^{16}\right.$ & $6.617 \times 10^{15}$ \\
$\Gamma_{1}\left(\mathrm{rad} \mathrm{s}^{-1}\right)$ & $5.09 \times 10^{16}$ & $1.7415 \times 10^{15}$ \\
$A_{2}\left(\mathrm{rad}^{-1}\right)$ & 679.7606 & 5377.4512 \\
$\phi_{2}\left(\mathrm{rad} \mathrm{s}^{-1}\right)$ & -0.0998 & -0.0092 \\
$\Omega_{2}\left(\mathrm{rd} \mathrm{s}^{-1}\right)$ & $3.4587 \times 10^{14}$ & $1.3545 \times 10^{14}$ \\
$\Gamma_{2}$ & $3.064 \times 10^{13}$ & $6.56505 \times 10^{12}$ \\
$A_{3}\left(\mathrm{rad}^{-1}\right)$ & 3.5244 & 2.6077 \\
$\Omega_{3}(\mathrm{rad} \mathrm{s}$ & -2.8539 \\
$\left.\Gamma_{3}(\mathrm{rad} \mathrm{s})^{-1}\right)$ & 4.6586 & $8.1007 \times 10^{14}$ \\
\hline$\Phi$ & $3.5832 \times 10^{15}$ & $8.7193 \times 10^{12}$ \\
\hline $\mathrm{L} 4$ & $1.68784 \times 10^{15}$ & 18.1032 \\
\hline$\Phi$ & 15.3895 & $\mathrm{Ag}$ \\
\hline
\end{tabular}

can use the CP3 model to simulate the plasmonic properties of highly tunable metallic nanostructures in a wide wavelength range between $180 \mathrm{~nm}$ and $2000 \mathrm{~nm}$ more precisely and easier (less memory and computing time) than the L4 model.

In Fig. 1, we compare the L4 and CP3 models with experimental data for gold. For the real part of the permittivity of gold, the L4 and CP3 models are both close to the experimental data, with a better fit for the CP3 model near $1900 \mathrm{~nm}$. For the imaginary part of permittivity of gold, the L4 and CP3 models give a similar description of the interband transitions of gold. Since the plasmon resonance wavelength is determined by the real part of the permittivity, it is more important to get a better fitting with the real part of the permittivity than the imaginary part of the permittivity in the spectrum. In Fig. 2, we compare the L4 and CP3 models with experimental data for silver. For the real part of the permittivity of silver, the $\mathrm{CP} 3$ model gives a much better fit with experimental data for wavelengths between $1200 \mathrm{~nm}$ and $2000 \mathrm{~nm}$, and the L4 model starts to deviate slightly from the experimental data at $1200 \mathrm{~nm}$. For the imaginary part of the permittivity of silver, the CP3 model behaves obviously better than the L4 model in the spectrum. The negative imaginary part of L4 model in some parts of the spectrum will result in an artificially near field enhancement in plane wave studies. Although the CP3 model is not very close to the experimental data, the values of the CP3 model are at least positive unlike the L4 model for silver.

In order to compare the two models more clearly, we define the fitness function $\Phi$ to compute the errors quantitatively for the wavelengths between $180 \mathrm{~nm}$ and $2000 \mathrm{~nm}$

$$
\Phi=\sum_{\omega}\left|\varepsilon_{\text {tab }}(\omega)-\varepsilon_{\text {model }}(\omega)\right|^{2}
$$

where $\varepsilon_{\text {tab }}(\omega)$ are the values tabulated from [3], and the sum is over the tabulated frequency values in [3]. The values of the fitness function of L4 and CP3 models are displayed in Table 1. For gold, the value of the fitness function of CP3 is smaller than half of that of the L4 model. For silver, the value of the fitness function of CP3 is less than that of the L4 model by an order of magnitude. From the above comparisons, we can judge that the CP3 model plus the fitting parameters displayed in Table 1 can be used in studying the optical properties of gold and silver more efficiently and accurately than the L4 model. In order to show that the CP3 model can be applied in the FDTD simulation, we simulate the extinction spectrum of a nanocylinder with a diameter of $40 \mathrm{~nm}$. In the inset of Fig. 3, the extinction spectra of a gold nanocylinder with a diameter of $40 \mathrm{~nm}$ using both the FDTD simulation and the Mie 


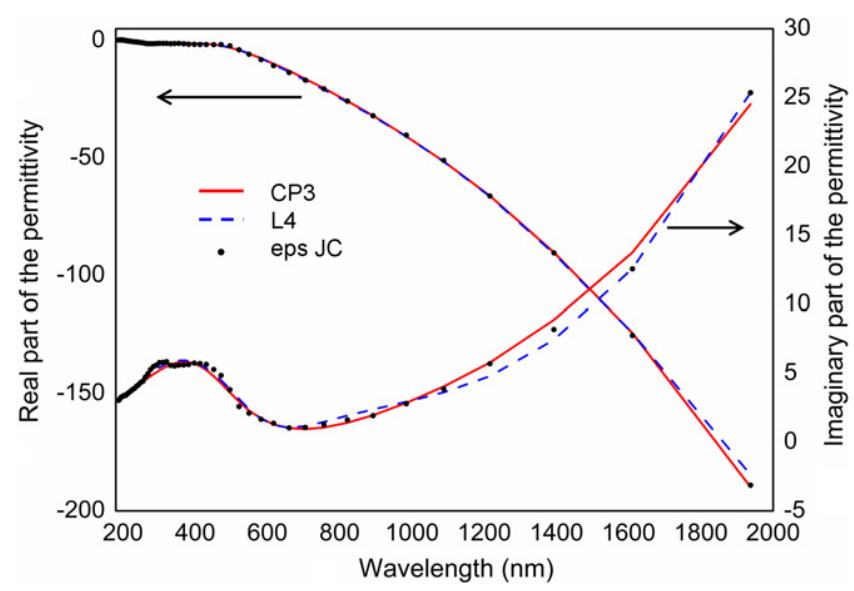

Fig. 1. (a) The permittivity of gold obtained from tabulated data (dots) [3], the CP3 model (dash blue lines), and the L4 model (red line). (For interpretation of the references to colour in this figure legend, the reader is referred to the web version of this article.)

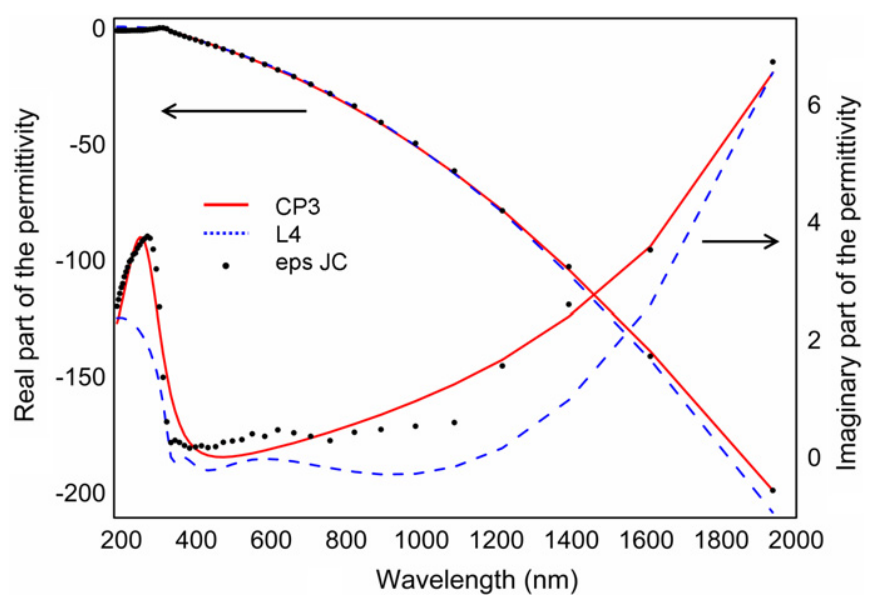

Fig. 2. (a) The permittivity of silver obtained from tabulated data (dots) [3], the CP3 model (dash blue lines), and the L4 model (red line). (For interpretation of the references to colour in this figure legend, the reader is referred to the web version of this article.)

theory [6] with the CP3 model show a good agreement. From this validation, we conclude that the CP3 model can be implemented into FDTD and the FDTD program we developed is accurate.

\section{Results and discussions}

Our FDTD simulation domain is separated into three regions from outside to inside: Absorbing Boundary, Scattered Field Region, and the Total Field Region. The perfect medium layers (PML) are used as absorbing boundary to prevent reflections of scattered waves back into the Total field. The FDTD calculations were done using a mesh size $0.4 \mathrm{~nm}$, a time step of $6.6697 \mathrm{e}-19 \mathrm{~s}$, and a Courant number of 0.5. The CP3 model was then incorporated into 2D-FDTD method to simulate the optical response of a pair of silica-core gold-shell nanocylinders with variable gap width for the polarization direction parallel to the axis of the pair. In the simulation, the outer and inner diameters of the core-shell nanocylinder is set at $80 \mathrm{~nm}$ and $50 \mathrm{~nm}$, respectively, the permittivity of the silica is 2.1 , and 


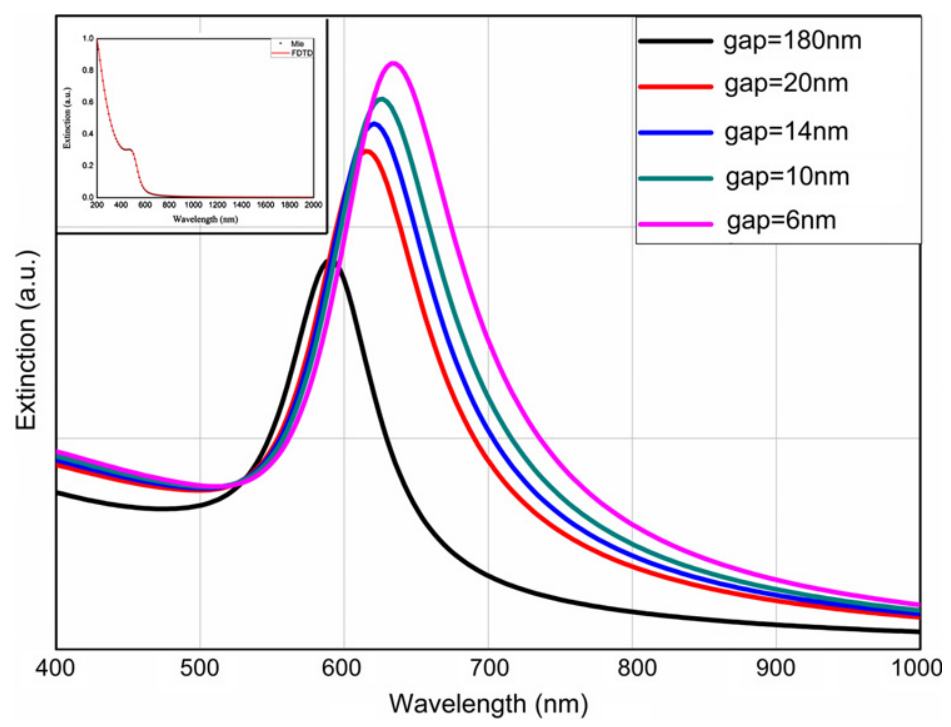

Fig. 3. The dependence of the extinction spectrum of a pair of core-shell nanocylinders on the gap width between the nanocylinders. The plasmon resonance wavelength shifts from $590 \mathrm{~nm}$ to $632 \mathrm{~nm}$ when the gap width decreases from $180 \mathrm{~nm}$ to $6 \mathrm{~nm}$. Inset: The extinction spectrum of a gold nanocylinder with a diameter of $40 \mathrm{~nm}$ obtained from the Mie theory (dots) and FDTD method (red lines) with the CP3 model. (For interpretation of the references to colour in this figure legend, the reader is referred to the web version of this article.)

the permittivity of gold material is described by the CP3 model with the parameters given in Table 1. In the simulation, the propagating direction of the short EM pulse is perpendicular to the axis of the pair and electric field of the EM wave is parallel to the axis of the pair. In order to perform spectral analysis, we keep running the discrete Fourier transforms along the total field and scattering field boundary (TFSF boundary). In Fig. 3, the simulation results show that due to the coupling between the core-shell nanocylinders when the gap width between the nanocylinders decreases, the resonance wavelength of the localized surface plasmon mode shifted to lower energy and the extinction efficiency increases. When the gap width decreases from $180 \mathrm{~nm}$ to $6 \mathrm{~nm}$, the plasmon resonance wavelength shifted from $590 \mathrm{~nm}$ to $632 \mathrm{~nm}$. These plasmon resonances are resulted from the dipole-dipole interaction between the nanocylinders and the dipole mode of the core-shell nanocylinder oscillates in-phase with another (the dipole mode is symmetric binding mode whose electrons at outer surface is aligned symmetrically with the electrons at inner surface [7]). We do not see the out-of-phase dipole-dipole interaction mode because the mode which does not have dipole moment cannot be induced by external light. Because the phase retardation effect need not be considered in such a size smaller than $1 / 4$ wavelength, there are no higher multipole modes in the extinction spectrum. In addition to changing the gap width, the ratio of the outer radius to inner radius of the core-shell nanocylinder, or the refractive index of the core can also be used to tune the resonance frequency.

\section{Summary}

An efficient dielectric function called CP3 model was developed and it was compared with the L4 model in the wavelength range between $180 \mathrm{~nm}$ and $2000 \mathrm{~nm}$. The results show that the CP3 model can be used in studying the optical properties of gold and silver more efficiently and accurately than the L4 model. The CP3 model was then incorporated into FDTD to study the coupling of the silica-core gold-shell nanocylinder pair. The simulation result shows that for the polarization direction parallel to the axis of the pair, the plasmon wavelength in the plasmon extinction maximum is red-shifted from $590 \mathrm{~nm}$ to $632 \mathrm{~nm}$ when the edge-to-edge gap decreases from $180 \mathrm{~nm}$ to $6 \mathrm{~nm}$. 


\section{Appendix}

For the stimulation window, $\Delta x$ is the spatial size and $\Delta t$ is the temporal time. In order to implement the CP3 model into FDTD method, we introduce the new vector field $\Psi_{C P}$ known as the recursive accumulators and the update equations become

$$
\begin{aligned}
& \left.\vec{\Psi}_{C P 1}\right|^{n}=\left.C_{c p 1}^{\rho} \vec{\Psi}_{C P 1}\right|^{n-1}+\left.C_{c p 1}^{\delta} \vec{E}\right|^{n} \\
& \left.\vec{\Psi}_{C P 2}\right|^{n}=\left.C_{c p 2}^{\rho} \vec{\Psi}_{C P 2}\right|^{n-1}+\left.C_{c p 2}^{\delta} \vec{E}\right|^{n} \\
& \left.\vec{\Psi}_{C P 3}\right|^{n}=\left.C_{c p 3}^{\rho} \vec{\Psi}_{C P 3}\right|^{n-1}+\left.C_{c p 3}^{\delta} \vec{E}\right|^{n} \\
& \left.E\right|^{n+1}=\left.C^{\alpha} \vec{E}\right|^{n}+C^{\beta} \nabla \times\left.\vec{H}\right|^{n+1 / 2}+C^{\gamma} \operatorname{Re}\left(\left.\vec{\Psi}_{C P 1}\right|^{n}+\left.\vec{\Psi}_{C P 2}\right|^{n}+\left.\vec{\Psi}_{C P 3}\right|^{n}\right) \\
& \left.\vec{H}\right|^{n+3 / 2}=\left.\vec{H}\right|^{n+1 / 2}+\frac{\Delta t}{\mu_{0} \Delta x} \nabla \times\left.\vec{E}\right|^{n+1} .
\end{aligned}
$$

The coefficients in Eqs. (A.1)-(A.3) are defined as

$$
\begin{aligned}
& C_{c p m}^{\rho}=-\mathrm{i} \frac{2 A_{m} \Omega_{m} \mathrm{e}^{\left(-\mathrm{i} \phi_{m}\right)}}{\Gamma_{m}-\mathrm{i} \Omega_{m}}\left(1-\mathrm{e}^{\left(-\Gamma_{m}+\mathrm{i} \Omega_{m}\right) \Delta t}\right)^{2} \quad m=1 \sim 3 \\
& C_{c p m}^{\delta}=\mathrm{e}^{\left(-\Gamma_{m}+\mathrm{i} \Omega_{m}\right) \Delta t} \quad m=1 \sim 3 .
\end{aligned}
$$

The values of the constants $A_{m}, \Omega_{m}, \Gamma_{m}, \phi_{m}$, and $\sigma$ whose physical meanings are amplitude, energy of the gap, broadening, phase, and conductivity can be found in Table 1 . The coefficients $C^{\alpha}, C^{\beta}$, and $C^{\gamma}$ used in Eq. (A.4) are defined as

$$
\begin{aligned}
C^{\alpha} & =\frac{\varepsilon_{\infty}}{\varepsilon_{\infty}+\chi_{0}} \\
C^{\beta} & =\frac{\Delta t}{\Delta x \varepsilon_{0}\left(\varepsilon_{\infty}+\chi_{0}\right)} \\
C^{\gamma} & =\frac{1}{\varepsilon_{\infty}+\chi_{0}}
\end{aligned}
$$

where $\varepsilon_{0}$ is the permittivity of free space, $\mu_{0}$ is the magnetic permeability of free space, and

$$
\chi_{0}=-\sigma \Delta t+\operatorname{Re}\left[\sum_{m=1}^{3}-\mathrm{i} \frac{A_{m} \Omega_{m} \mathrm{e}^{-\mathrm{i} \phi_{m}}}{\Gamma_{m}-\mathrm{i} \Omega_{m}}\left(1-\mathrm{e}^{\left(-\Gamma_{m}+\mathrm{i} \Omega_{m}\right) \Delta t}\right)\right] .
$$

\section{References}

[1] F. Hao, P. Nordlander, Chem. Phys. Lett. 446 (2007) 115.

[2] A. Vial, T. Laroche, Appl. Phys. B 93 (2008) 139.

[3] P. Johnson, R. Christy, Phys. Rev. B 6 (1972) 4370.

[4] A. Taflove, S.C. Hagness, Computational Electrodynamics: The Finite-Difference Time-Domain Method, 3rd ed., Artech House, Boston, 2005.

[5] A. Vial, J. Opt. A: Pure Appl. Opt. 9 (2007) 745.

[6] C.F. Bohren, D.R. Huffman, Absorption and Scattering of Light by Small Particles, John Wiley \& Sons Inc., New York, 1998.

[7] E. Prodan, C. Radloff, N.J. Halas, P. Norlander, Science 302 (2003) 419. 